\title{
A Corpus-based Study of English Translation of Terminology in Traditional Chinese Medicine
}

\author{
Cheng Liu ${ }^{1, a}$ and Xiaofang Wang ${ }^{2, b^{*}}$ \\ School of Humanities, Jiangxi University of TCM, No. 1688 of Meiling Road, Wanli District, \\ Nanchang City, Jiangxi Province, China \\ School of Humanities, Jiangxi University of TCM, No. 1688 of Meiling Road, Wanli District, \\ Nanchang City, Jiangxi Province, China \\ ajxjzlc@163.com, b920711622@qq.com \\ * The Corresponding Author
}

\section{Keywords: Corpus-based; English translation; Terminology; Traditional Chinese medicine}

\begin{abstract}
Term translation of TCM is critical to the success of international communication of TCM culture. Researchers and scholars have been exploring the translation of TCM terms from different perspectives and attempting to establish unified standards for the translation of TCM terms. The corpus-based practice of TCM term translation investigates into the differences and similarities of TCM term translation home and abroad, trying to come up with the basic principles and approaches for the translation of TCM terms, in the hope of providing more subjective and comprehensive reference and norms for the translation of TCM terms.
\end{abstract}

\section{Research Status of Translation of Traditional Chinese Medicine}

In order to enhance the international communication of traditional Chinese medicine, the scholars of the medical and foreign language circles have made unremitting efforts to study the translation of traditional Chinese medicine. At present, researchers have explored the translation of nouns and terminology from different angles, trying to establish the standard of the unification of traditional Chinese medicine. First of all, they discussed the translation features of Chinese scholars, and put forward various Chinese translation principles, for example, scientific principles, the information principle, standardization principle, accepted principle, culture principle, back translation principle, when translation, equivalent translation and transliteration ${ }^{[1]}$. Secondly, under the guidance of translation theory, many researchers have discussed the strategies and methods of TCM translation, such as transliteration and literal translation, synthesis, domestication and foreignization, simplification, morpheme translation and so on. ${ }^{[2]}$ However, due to the particularity of TCM literature, there are significant differences between Chinese medicine and Western medicine in terms of system and way of thinking. There are still several unsolved problems in English translation of traditional Chinese medicine, because of different understanding of physiology and pathology. The first is the lack of corresponding medical terms. Because of the great difference between Chinese medicine and Western medicine, many TCM terms lack corresponding western medicine terms. Although some of them are similar or even identical, their connotations are quite different. Therefore, it is difficult to find exact terminology translation. In addition, many terms in traditional Chinese medicine lack the same character, which also brings difficulties to translation work. Second, from a linguistic perspective, ancient Chinese literati are good at using various rhetorical devices to achieve the best expression effect. Therefore, Chinese literature has been given a lot of figures of speech, and the difficulty of translation is increasing. Third, the multidisciplinary nature of Chinese traditional medicine, especially the connection with traditional philosophy, embodies the characteristics of the blend of medicine and philosophy. The cultural connotation between the lines of Chinese medicine and the problem of solving the literature of Chinese medicine are the obstacles in understanding the translation of traditional Chinese medicine. Finally, the correspondence of TCM translation refers to the correspondence of word meaning rather than the correspondence of words. To achieve such a correspondence, it is bound to add explanatory translation, which will violate the conciseness of translation. Because a considerable number of 
Chinese medicine translators lack the background of traditional Chinese medicine, many terminology concepts are not clear and translation lacks unified standards. In view of the fact that there are still many unsolved problems in the translation of TCM English at home and abroad, we will continue to do the research in this field. At present, the domestic and foreign research on the English translation of traditional Chinese medicine and some single Chinese medicine terminology translation (for example, yin and Yang, five elements, energizer, shenming's translation), some research on a translation of TCM terms (such as the translation of prescription, pulse condition, title of TCM classics, Chinese traditional philosophy terminology, four-character term, etc.).

Corpus linguistics, which has been rising in the past twenty years, has provided a new method for the study of language and translation. The researchers collected a large number of authentic materials and built various types of corpus for the use of researchers from different academic backgrounds. Corpus linguistics not only has the advantages of American structural linguistics, but also has the advantage of the Chomsky school's introspection method ${ }^{[3]}$. It is highly valued by the researchers for its representativeness, authenticity and the objectivity of the analysis. Some scholars have pointed out that corpus is becoming the mainstream. It has become the default data source in language research. The highest expectation in linguistics circles is closely related to corpus linguistics.

Corpus based translation study developed in recent years is a new research paradigm in translation studies. On the basis of contemporary linguistic theory, it makes use of modern means such as probability and statistics to study the diachronic or synchronic translation of bilingual real corpus. The development of corpus makes the descriptive translation study be fully developed. In recent years, the research on the universal characteristics of translation based on corpus has been mainly tested on the hypothesis of Baker from three aspects: simplification, visualization and generalization. The researchers explored the features of the translated text relative to the original text.

This kind of research usually embodies the combination of theoretical interpretation and empirical research. Compared with the general characteristics of corpus based translation, domestic research is still at the theoretical level. The empirical research on the universal characteristics of the newly started corpus is mainly focused on the study of Chinese translation language. The researchers based on the bilingual corpus to explore the characteristics of Chinese translation, English translation of TCM terms there are many different versions in the translation of medical circles, various words have their own basis, but this area that has been the lack of a unified standard can be recognized by most scholars at home and abroad. The Chinese and foreign scholars, each one sticks to his own view to reach a consensus of the situation is not conducive to the traditional Chinese medicine to the world. The establishment of TCM terminology corpus, researchers, through a large number of examples, can conclude the expression characteristics and the more complete observation of English translation of TCM terms systematically the statistical expression form, on the basis of quantitative analysis and qualitative analysis, more objectively fully reveal the characteristics of English translation of TCM terms and rules, establish better TCM terms of norms and standards to further improve the theoretical system of TCM English, and lay a foundation for foreign language communication in medicine and Chinese culture. So far, there is no established and available TCM English corpus. However, many scholars have discussed the feasibility, principles, methods and significance of building corpus of TCM English. ${ }^{[5]}$ Based on the practice of C-E terminology translation, based on the exploration of the differences and similarities between Chinese and foreign English translation of TCM terms, we summarize the basic principles and methods of TCM term translation, and provide a more objective and comprehensive reference standard and basis for the standardization of TCM translation.

\section{Necessity and Feasibility of Building an English Corpus of Traditional Chinese Medicine}

In recent years, the research of English translation of traditional Chinese medicine is mainly from the traditional point of view. Only a few scholars turn their eyes to the research method of corpus. Corpora rely on powerful computer technology, making it possible to quantify the translation of 
descriptive translation, which can greatly promote the development of English translation of traditional Chinese medicine. In particular, it can promote the translation of traditional Chinese medicine terms, speed up the standardization of the development of traditional Chinese medicine terms, and make traditional Chinese medicine go to the world as soon as possible. At present, Chinese universities have not yet built a large TCM English corpus. The authors hope to promote the development of TCM translation by building a bilingual parallel corpus. The corpus should be reasonably determined according to the purpose of building the corpus and the attributes of the corpus, not the greater the better. Specifically, the bilingual corpora of TCM terms belong to the corpus of special purpose, and the scale does not need large corpora, such as the general English corpus, such as the British National Corpus. The construction of the corpus of basic terminology of traditional Chinese medicine includes 4 authoritative English translation standards. It will be gradually perfected in use and then extended to other English translation standards. It is clear that it is feasible to build the bilingual parallel corpus of the basic nouns of traditional Chinese medicine, and it can also promote the study of English translation in traditional Chinese medicine.

\section{The Establishment of English Bilingual Parallel Corpus for the Basic Terminology of Traditional Chinese Medicine}

Parallel corpora of TCM will greatly promote the development of TCM English translation, but the workload of establishing parallel corpus is huge. Special computer software also discourages foreign language teachers. We hope that the self-built small corpus can promote the development of Chinese English translation research.

Before establishing the corpus of English synonyms in the basic terminology of traditional Chinese medicine, we first examine the Chinese synonyms of the terminology of traditional Chinese medicine. We chose more authoritative Chinese reference books, such as Dictionary of TCM, Collection of TCM Words, check the words one by one. Generally speaking, in the same TCM tool book, a group of synonyms will be interpreted by the editor to choose one of them as a positive name (the standard name). The other synonyms link to the positive name.

During the course of collecting of words, we use the official term as the standardized one, and other callings will also be listed in the interpretation of the official term. In the collection of English synonyms, the corresponding Chinese terms in the two dictionaries. When we processed basic terms in the four standards of Terminology of Traditional Chinese medicine, WHO International Standard Terminologies name on Traditional Medicine in the Western Pacific Region, Chinese-English international standards of basic terminology of Traditional Chinese Medicine, A PRACTICAL DICTIONARY OF CHINESE MEDICINE, we can get the collection of English synonyms related with the official Chinese terms.

In the process of collecting terms, computer assistance is needed, and more manual control is needed to reduce the error of input. For the above four translation standards of TCM nomenclature, the research group already has the electronic version, after designing the demo program; we can properly grasp the corresponding entries to ensure the smooth construction of corpus. The results of computer processing need to be audited manually, and if there is a problem, the corresponding modification will be given. The application of computer technology and the combination of manual work will make the textual research and corpus construction more complete and accurate.

English synonym corpus of basic nomenclature of Chinese medicine involves mainly involves four parts: the Basic Theory of Traditional Chinese Medicine (804 entries), Chinese Medicine Diagnosis (930 entries), Materia Medica(641 entries), Pharmacy Prescription (559 entries), which were included in above mentioned four standards. The English synonyms of TCM terms will be classified and put together, and be easily stored with a corpus management system. We eventually make the English synonym corpus of basic terms of Traditional Chinese Medicine. After examined by the relevant experts, the corpus will be linked to the campus net, so that Chinese researchers and translators can retrieve the standardized terms, English synonyms and definitions of basic TCM terminology. 


\section{Conclusion}

Through researching the English translation entries of texts with TCM terms, we can find differences and similarities between Chinese and foreign English translations of TCM terms, summarize the basic principles of translation of TCM terms, and will further deepen the study of the general characteristics of translation. Meanwhile, corpus based study of TCM term translation can provide more empirical evidence for standardization of TCM terms and provide a more objective and comprehensive reference standard for standardization of TCM translation.

\section{Acknowledgements}

Research Project of Humanities and Social Science for Colleges and Universities in Jiangxi Province (No: YY162002); Research of Education Science Planning Projects of $13^{\text {th }}$ Five-Year in Jiangxi Province (No: 17YB145); Key Project of Teaching Reform of Jiangxi University of Traditional Chinese Medicine (No: 2016jzzd-1).

\section{Reference}

[1] Xu Yonghong. Research on the external communication of traditional Chinese medicine culture -- A Perspective of cultural adaptation [D]. Shanghai: East China Normal University, 2014:83-85.

[2] Zhou Chuan. The current situation and Reflection of Chinese traditional Chinese medicine culture in overseas development [J]. World Magazine of traditional Chinese and Western medicine, 2011 (8): 733.

[3] Ma Boying. The perplexity of the Chinese English Translation: how the meaning and context accurately express the English translation of Chinese traditional Chinese medicine ([J]. Global Traditional Chinese Medicine, 2012,5 (11): 848-851.

[4] Xu Chunjie, Zhao Qiurong. The Chinese translation framework of parallel corpus research [J]. Foreign Language Journal, 2014 (4): 152-154.

[5] Zhang Ping. Application of Chinese-Japanese bilingual parallel corpus in translation teaching of Chinese Medicine Nursing -- Taking Plains as the center [J]. Journal of Chengdu University of Traditional Chinese Medicine (SOCIAL SCIENCE EDITION), 2015, 17 (2): 61-62. 